\title{
Connectedness at infinity of systolic complexes and groups
}

\author{
Damian Osajda*
}

\begin{abstract}
By studying connectedness at infinity of systolic groups we distinguish them from some other classes of groups, in particular from the fundamental groups of manifolds covered by Euclidean space of dimension at least three. We also study semistability at infinity for some systolic groups.
\end{abstract}

Mathematics Subject Classification (2000). 20F65, 20F67, 20F69, 57M07.

Keywords. Simplicial non-positive curvature, topology at infinity.

\section{Introduction}

Systolic complexes were introduced by T. Januszkiewicz and J. Świątkowski ([JS1]) and, independently, by F. Haglund ([H]) as combinatorial analogues of nonpositively curved spaces. They are simply connected simplicial complexes satisfying some local combinatorial conditions: roughly speaking there is a lower bound for the lengths of "essential" closed paths in the one-skeleton of every link.

This condition is an analogue of the Gromov condition implying nonpositive curvature for cubical complexes. However systolic complexes equipped with the metric for which every simplex is isometric to the regular euclidean simplex are not necessarily nonpositively curved. Conversely, there exist nonpositively curved spaces (e.g. manifolds of dimension at least three) that do not admit systolic triangulations. Nevertheless systolic spaces (sometimes referred to as complexes of simplicial nonpositive curvature - SNPC) possess many properties analogous to those of spaces of non-positive curvature (we use [JS1] as a reference for this theory). They are contractible (this is an analogue of the Cartan-Hadamard theorem), with some additional assumptions they are Gromov hyperbolic or CAT(0) (CAT $(-1))$, and complexes of groups with links satisfying the same conditions as links in systolic spaces are developable.

${ }^{*}$ The author is a fellow of the Marie Curie Intra-European Fellowship, contract MEIF CT2005 010050, and was partially supported by the Polish Scientific Research Committee (KBN) grant 2 P03A 01725. 
The latter property allows one to construct many examples of systolic spaces and groups (i.e. groups acting geometrically on systolic complexes) with some additional properties and to answer some open questions.

Systolic groups are biautomatic ([JS1]). They can be considered as higher dimensional analogues of small cancelation groups ([W]). Ideal boundaries of 7-systolic groups are strongly hereditarily aspherical compacta ([O]).

We study the topology at infinity of systolic complexes and groups. It turns out that connectedness at infinity allows to distinguish systolic groups from some other classes of groups, in particular from fundamental groups of closed manifolds covered by $\mathbb{R}^{n}$, for $n>2$ (see Section 3). This extends some of the results obtained in [JS2] and [W] (see Subsection 3.1 below). Our main tool is the property $\operatorname{Conn}_{k}^{\infty}(G)$ (see Section 2.3) saying that for every contractible, rigid $G$-CW complex $X$ on which $G$ acts properly discontinuously and cocompactly and for every neighborhood $U$ of infinity in $X$ there exists a smaller neighborhood of infinity $V \subset U$ such that every $k$-dimensional sphere in $V$ is homotopically trivial in $U$. The main result is the following.

Theorem 1 (Theorem 3.1 in Section 3). Let $X$ be a locally finite systolic complex, let $G$ be a systolic group and let $k \geq 2$ be a natural number. Then the conditions $\operatorname{Conn}_{k}^{\infty}(X)$ and $\operatorname{Conn}_{k}^{\infty}(G)$ hold.

As a corollary of this theorem, using some homological algebra, we obtain the following.

Theorem 2 (Theorem 3.2 in Section 3). Systolic groups are not simply connected at infinity.

In Subsection 3.1 we give some examples of groups that are not systolic. Among them there are some Coxeter groups, lattices in isometry groups of some buildings and nonpositively curved cubical complexes.

In Section 4 we study topology at infinity of locally finite systolic chamber complexes such that links of vertices are connected and complements of open balls of radius 2 in those links are connected - condition $R(v, X)$ in Section 4. Our results can be viewed as analogues of some of the results obtained in $[\mathrm{BMcCM}]$ for CAT(0) complexes and in $[\mathrm{DM}]$ for Coxeter groups. We prove the following.

Theorem 3 (Theorem 4.6, Section 4). Let $X$ be a locally finite systolic chamber complex of dimension greater than one. Suppose that $R(v, X)$ holds for almost every vertex $v \in X$ (i.e. for all but finitely many). Then $X$ is not simply connected at infinity. Moreover, if $G$ acts properly and cocompactly on $X$ then $G$ is semistable at infinity.

As shown in Section 4 for a systolic group acting on a normal pseudomanifold $X$ the condition $R(v, X)$ holds for every vertex $v$. Such groups of arbitrarily large 
cohomological dimension are constructed in [JS1]. Those groups are the only systolic groups of cohomological dimension greater than two known to us at the moment.

Semistability at infinity is conjectured to hold for all one ended finitely presented groups. Thus we just give new examples of groups for which it holds.

I would like to thank Tadeusz Januszkiewicz and Jacek Świątkowski for introducing me to the subject and suggesting the problem. I am also grateful to them and to Jan Dymara, Ross Geoghegan, Peter Kropholler, Ian Leary and Carrie Schermetzler for helpful conversations.

\section{Preliminaries}

2.1. Systolic complexes and groups. A simplicial complex $X$ is flag if every finite subset of its vertices pairwise connected by edges spans a simplex of $X$. Following [JS1] and [JS2] we say that, for a given natural $k \geq 4$, a simplicial complex $X$ is $k$-large if it is flag and if every cycle $\gamma$ in $X$ (i.e. its subcomplex homeomorphic to the circle) of length $4 \leq|\gamma|<k$ has a diagonal (i.e. an edge connecting two nonconsecutive vertices in $\gamma$ ). Here $|\gamma|$ denotes the number of edges of $\gamma$.

A simplicial complex $X$ is locally $k$-large if for every simplex $\sigma \neq \emptyset$ of $X$ its link $X_{\sigma}$ in $X$ is $k$-large.

$X$ is $k$-systolic if it is locally $k$-large, connected and simply connected.

Because $k=6$ is of special importance in that theory, 6-systolic complexes are called systolic.

A group acting geometrically (i.e. properly discontinuously and cocompactly) by simplicial automorphisms on a $k$-systolic (resp. systolic) complex is called $k$-systolic (resp. systolic).

For example free groups and fundamental groups of surfaces are systolic. For other examples see below.

In the rest of this subsection we list some results concerning systolic complexes and groups which we will use later. If it is not stated otherwise they can be found in [JS1] and we follow the notation of that paper. In particular, for a simplicial complex $X$ we denote by $X^{(i)}$ its $i$-skeleton, and we denote by $\sigma * \rho$ the join of simplices $\sigma$ and $\rho$.

Recall that a subcomplex $Y$ of a simplicial complex $X$ is full if every set $A$ of vertices of $Y$ spanning a simplex of $X$ spans a simplex in $Y$. The following facts are immediate:

Proposition 2.1. (1) A full subcomplex in a (locally) $k$-large complex is (locally) $k$-large.

(2) Links of a $k$-large complex are $k$-large.

(3) ([JS1], 1.8.5) There is no $k$-large triangulation of the 2-sphere for $k \geq 6$. Hence no triangulation of a manifold of dimension $n \geq 3$ is 6-large since 2-spheres would occur as links of some simplices in such triangulation. 
Now let $X$ be a systolic complex and $\sigma$ its simplex. By Section 7 of [JS1] one can define a closed combinatorial ball of radius $i$ around $\sigma$ in $X, B_{i}(\sigma, X)$, inductively: $B_{0}(\sigma, X)=\sigma$ and $B_{i}(\sigma, X)=\bigcup\left\{\tau \mid \tau \cap B_{i-1}(\sigma, X) \neq \emptyset\right\}$ for $i>0$. (Note that in Section 7 of [JS1] the combinatorial balls and the deformation retractions we consider are defined more generally for any convex subcomplex of $X$.)

By $S_{i}(\sigma, X)$ we denote the subcomplex of $B_{i}(\sigma, X)$ spanned by the vertices at combinatorial distance $i$ from $\sigma$, i.e. not belonging to $B_{i-1}(\sigma, X)$. By $\stackrel{\circ}{B}_{i}(\sigma, X)$ we denote the interior of the closed combinatorial $i$-ball around $\sigma$ in $X$, i.e., $\stackrel{\circ}{B}_{i}(\sigma, X)=$ $B_{i}(\sigma, X) \backslash S_{i}(\sigma, X)$.

Thus one can define closed combinatorial balls of small radii in $k$-large complexes and they are isomorphic to ones in the corresponding universal covers. In the following lemma the first claim implies the second one in view of Fact 2.1.

Proofs of the following two lemmas (except the last part of the second lemma) can be found in Section 7 of [JS1].

Lemma 2.2. The ball $B_{i}(\sigma, X)$ and the sphere $S_{i}(\sigma, X)$ are full subcomplexes of $X$ and they are $k$-large.

Lemma 2.3. For every simplex $\tau \in S_{i}(\sigma, X), i>0, \rho=S_{i-1}(\sigma, X) \cap X_{\tau}$ is a single simplex and $X_{\tau} \cap B_{i}(\sigma, X)=B_{1}\left(\rho, X_{\tau}\right)$ and $X_{\tau} \cap S_{i}(\sigma, X)=S_{1}\left(\rho, X_{\tau}\right)$.

Proof. We prove here only that $X_{\tau} \cap S_{i}(\sigma, X)=S_{1}\left(\rho, X_{\tau}\right)$.

Let a vertex $v$ belong to $X_{\tau} \cap S_{i}(\sigma, X)$. Then, since $\rho \subset B_{i-1}(\sigma, X)$ we have $v \in S_{1}\left(\rho, X_{\tau}\right)$ and hence $X_{\tau} \cap S_{i}(\sigma, X) \subset S_{1}\left(\rho, X_{\tau}\right)$.

Now let a vertex $v$ belong to $S_{1}\left(\rho, X_{\tau}\right)$. Then it is obvious that $v \in S_{i}(\sigma, X) \cup$ $S_{i-1}(\sigma, X)$. Assume $v \in S_{i-1}(\sigma, X)$. Then $v * \rho \in S_{i-1}(\sigma, X) \cap X_{\tau}=\rho$ which is a contradiction. Hence $X_{\tau} \cap S_{i}(\sigma, X) \supset S_{1}\left(\rho, X_{\tau}\right)$.

In the rest of the paper we call the simplex $\rho$, as in the above lemma the projection of $\tau$ on $B_{i-1}(\sigma, X)$.

For a simplicial complex $X$, we denote by $X^{\prime}$ its first barycentric subdivision. For a simplex $\sigma \in X$ we denote by $b_{\sigma}$ the barycenter of $\sigma$.

By the lemma above we can define an elementary contraction

$$
\pi_{B_{i}(\sigma, X)}: B_{i+1}(\sigma, X)^{\prime} \rightarrow B_{i}(\sigma, X)^{\prime}
$$

between barycentric subdivisions of balls by putting

$$
\pi_{B_{i}(\sigma, X)}\left(b_{v}\right)= \begin{cases}b_{v \cap B_{i}(\sigma, X)} & \text { if } v \cap B_{i}(\sigma, X) \neq \emptyset, \\ b_{X_{v} \cap B_{i}(\sigma, X)} & \text { if } v \cap B_{i}(\sigma, X)=\emptyset\end{cases}
$$

and then extending simplicially. In Section 8 of [JS1] it is shown that $\pi_{B_{i}(\sigma, X)}$ is a deformation retraction and that $\pi_{B_{i}(\sigma, X)}\left(B_{i+1}(\sigma, X) \backslash \stackrel{\circ}{B}_{i}(\sigma, X)\right) \subset S_{i}(\sigma, X)$. 
Then we define a deformation retraction $P_{B_{i}(\sigma, X)}: X \rightarrow B_{i}(\sigma, X)$ as follows. If $x \in B_{j}(\sigma, X)$ then $P_{B_{i}(\sigma, X)}(x)=\pi_{B_{i}(\sigma, X)} \circ \pi_{B_{i+1}(\sigma, X)} \circ \cdots \circ \pi_{B_{j-1}(\sigma, X)}(x)$.

Lemma 2.4. For $j>i$, the projection $\left.P_{B_{i}(\sigma, X)}\right|_{B_{j}(\sigma, X)}: B_{j}(\sigma, X) X \rightarrow B_{i}(\sigma, X)$ provides a deformation retraction of $B_{j}(\sigma, X) \backslash \stackrel{\circ}{B_{i}}(\sigma, X)$ onto $S_{i}(\sigma, X)$ within $B_{j}(\sigma, X) \backslash \stackrel{\circ}{B_{i}}(\sigma, X)$.

For $k \geq 6$, by Corollary 1.5 of [JS1] (compare Fact 2.1 above), a simplicial complex $Y$ is $k$-large if and only if it is locally $k$-large and the minimal length of a homotopically nontrivial (in $Y$ ) loop in the 1-skeleton $Y^{(1)}$ of $Y$ is at least $k$. Hence we obtain the following.

Lemma 2.5. Let $k \geq 6$ and let $Y$ be a $k$-large simplicial complex. If $p: X \rightarrow Y$ $i$ the universal cover of $Y$ and $m<\frac{k-1}{2}$ then for $i=0,1,2, \ldots, m$ the map $\left.p\right|_{B_{i}(\sigma, X)}: B_{i}(\sigma, X) \rightarrow p\left(B_{i}(\sigma, X)\right)$ is an isomorphism.

By part 3 of Fact 2.1 there is no systolic manifold above dimension two. But there are systolic groups acting geometrically on spaces that are "close" to manifolds as we describe below.

A simplicial complex $X$ is called a chamber complex of dimension $n$ if it is the union of its $n$-simplices (which are called chambers of $X$ ) and for every $(n-1)$ dimensional face of $X$ there exist at least two chambers containing it. It is easy to see that links in a chamber complex are themselves chamber complexes. A gallery in a chamber complex is a finite sequence of maximal simplices such that two consecutive simplices share a common face of codimension 1. A chamber complex is said to be gallery connected if any two chambers can be connected by a gallery. A chamber complex is normal if it is gallery connected and all its links of dimension above 0 are gallery connected. A chamber complex is a pseudomanifold if every codimension one face belongs to exactly two maximal simplices.

In [JS1] it is shown (Corollary 19.2) that for every natural $n$ and every $k \geq 6$ there exists an $n$-dimensional compact chamber complex that is $k$-large. Such examples are constructed as developments of some simplices of groups. Moreover, they can be pseudomanifolds. In Section 5 we show that the groups constructed this way are oneended and semistable at infinity. Here we give some background on the constructions given in [JS1].

Recall (we use here the notation of [JS1]; a standard reference for complexes of groups is $[\mathrm{BriH}])$ that for a given simplex $\Delta$ a simplex of groups $\mathcal{E}$ over $\Delta$ is a family of groups $\left\{G_{\sigma} \mid \sigma\right.$ is a subsimplex of $\left.\Delta\right\}$ together with the family of structure homomorphisms $\left\{\psi_{\sigma \tau}: G_{\sigma} \rightarrow G_{\tau} \mid \tau \subset \sigma\right\}$ satisfying natural commutation relations. A simplex of groups $\mathcal{E}$ is called developable if there exists a simplicial complex $X$ (development) and a group $G$ acting on $X$ such that $G \backslash X=\Delta$, where $\Delta$ is identified with some maximal simplex of $X, G_{\sigma}=\operatorname{Stab}(\sigma, G)$ for $\sigma$ being a subsimplex of $\Delta$ 
and $\psi_{\sigma \tau}$ are the inclusions $G_{\sigma} \rightarrow G_{\tau}$ for $\tau \subset \sigma$. We allow the case $\sigma=\emptyset$ and then we put $G_{\emptyset}=G$. Then we write $\mathscr{G}=G \backslash \backslash X$. We say also that $X$ is the development of $\mathscr{E}$ with respect to the morphism $m: \mathscr{G} \rightarrow G$. The morphism $m$ is the family of injections $m_{\sigma}: G_{\sigma} \rightarrow G$ agreeing with structure homomorphisms, for $\sigma$ being simplices of $\Delta$. Moreover, if $\mathcal{E}$ is developable then there exists a simply connected simplicial complex $\widetilde{\mathscr{G}}$ (universal development of $\mathscr{\mathscr { G }}$ ) and a group $\hat{\mathscr{G}}$ (direct limit of $\mathscr{\mathscr { G }}$ ) such that $\mathscr{\mathscr { E }}=\hat{\mathscr{E}} \backslash \widetilde{\mathscr{E}}$. For a subsimplex $\sigma$ of $\Delta$ one can consider a simplex of groups $\mathcal{E}^{\sigma}$ over a link $\Delta_{\sigma}$, being a "restriction of $\mathcal{E}$ to the link". There is a canonical morphism $i_{\sigma}: \mathscr{\ell}^{\sigma} \rightarrow G_{\sigma}$.

Following [JS1] for a natural $k \geq 4$ we will call a simplex of groups $\mathcal{E}$, locally $k$-large if for every link $\Delta_{\sigma}$, the development of $\mathcal{E}^{\sigma}$ with respect to the canonical morphism $i_{\sigma}: \mathscr{E}^{\sigma} \rightarrow G_{\sigma}$ is $k$-large.

Proposition 2.6 (Corollary 17.4 in [JS1]). For $k \geq 6$ every locally $k$-large simplex of groups is developable.

In Section 18 of [JS1] a notion of (local) extra-tilability of simplices of groups and of their developments is introduced. The most important for us is the following property.

Proposition 2.7 (Proposition 18.3 in [JS1]). Let $\mathscr{E}$ be a locally extra-tilable simplex of groups. Then the action of the direct limit $\hat{\mathscr{G}}$ on the universal development $\tilde{\mathscr{E}}$ has the following property: each $i$-ball $B_{i}(\sigma, \widetilde{\mathscr{E}})$, for any natural $i$, is a strict fundamental domain for the action of a unique subgroup of $\hat{\mathscr{E}}$.

By applying Proposition 2.7, it is shown in [JS1] that for given natural $k \geq 6$, simplex $\Delta$ and a family of finite groups $\left\{A_{s} \mid s\right.$ is a codimension 1 face of $\left.\Delta\right\}$ there exists a simplex of groups $\mathcal{G}$ over $\Delta$ such that $G_{s}=A_{s}$, for every codimension one face, and $\mathscr{E}$ admits finite $k$-large development. The explicit construction of local groups $G_{\sigma}$ of such $\mathcal{E}$ goes by induction on the codimension of $\sigma$. The important point is that for every $\sigma$, local developments of $\mathscr{E}$ at $\sigma$ are $k$-large and extra-tilable.

2.2. Homology of groups. The following two propositions and their proofs were supplied by Ian Leary. The main reference for homology of groups is [Bro1].

Proposition 2.8. If a group $G$ acts with finite stabilizers on a finite-dimensional contractible simplicial complex $X$ then:

(1) $\operatorname{cd}_{\mathbb{Q}} G \leq \operatorname{dim}(X)$.

(2) There exists a free $\mathbb{Q} G$-module $F=\bigoplus_{i} \mathbb{Q} G$ and $n^{\prime} \leq \operatorname{dim}(X)$ such that $H^{n^{\prime}}(G ; F) \neq 0$. 
(3) There exists a free $\mathbb{Z} G$-module $\widetilde{F}=\bigoplus_{j} \mathbb{Z} G$ and $n=n^{\prime}$ or $n=n^{\prime}+1$ such that $H^{n}(G ; \widetilde{F}) \neq 0$.

Proof. 1. Let $H$ be a finite subgroup of $G$ and let $\pi: \mathbb{Q} G \rightarrow \mathbb{Q} G / H$ be the projection map given by $\pi(g)=g H$. Then there is a section $s: \mathbb{Q} G / H \rightarrow \mathbb{Q} G$ of $\pi$, defined by $s(g H)=\frac{1}{|H|} \sum_{h \in H} g h$. Hence $\mathbb{Q}[G / H]$ is $\mathbb{Q} G$-projective and the simplicial chain complex $C_{*}(X ; \mathbb{Q})$ is a projective resolution of $\mathbb{Q}$ over $\mathbb{Q} G$ (compare Exercise I.8.5 in [Bro1]).

2. Since $\operatorname{cd}_{\mathbb{Q}} G \leq \operatorname{dim}(X)<\infty$, we have $\operatorname{cd}_{\mathbb{Q}} G=\max \left\{n^{\prime} \mid\right.$ there exists a $\mathbb{Q} G$-module $M$ with $\left.H^{n^{\prime}}(G ; M) \neq 0\right\}$.

Given such an $M$, let $F$ be a free $\mathbb{Q} G$-module fitting into the short exact sequence

$$
0 \rightarrow K \rightarrow F \rightarrow M \rightarrow 0
$$

The cohomology long exact sequence gives

$$
H^{n^{\prime}}(G ; F) \rightarrow H^{n^{\prime}}(G ; M) \rightarrow H^{n^{\prime}+1}(G ; K) .
$$

Since $H^{n^{\prime}+1}(G ; K)=0$ we have $H^{n^{\prime}}(G ; F) \neq 0$.

3 . There is a short exact sequence of abelian groups

$$
0 \rightarrow \bigoplus_{m \geq 1} \mathbb{Z} \rightarrow \bigoplus_{m \geq 1} \mathbb{Z} \rightarrow \mathbb{Q} \rightarrow 0
$$

Hence there is a short exact sequence of $\mathbb{Z} G$-modules

$$
0 \rightarrow \bigoplus_{m \geq 1} \mathbb{Z} G \rightarrow \bigoplus_{m \geq 1} \mathbb{Z} G \rightarrow \mathbb{Q} G \rightarrow 0
$$

and a short exact sequence of $\mathbb{Z} G$-modules

$$
0 \rightarrow \tilde{F} \rightarrow \tilde{F} \rightarrow F \rightarrow 0 .
$$

The cohomology long exact sequence gives

$$
H^{n^{\prime}}(G ; \widetilde{F}) \rightarrow H^{n^{\prime}}(G ; \widetilde{F}) \rightarrow H^{n^{\prime}}(G ; F) \rightarrow H^{n^{\prime}+1}(G ; \widetilde{F}) .
$$

Since $H^{n^{\prime}}(G ; F) \neq 0$ we obtain $H^{n^{\prime}}(G ; \widetilde{F}) \neq 0$ or $H^{n^{\prime}+1}(G ; \widetilde{F}) \neq 0$.

Proposition 2.9. If a group $G$ acts with finite stabilizers and cocompactly on a finitedimensional contractible simplicial complex $X$ then there exists a natural number $n \leq \operatorname{dim}(X)$ such that $H^{n}(G ; \mathbb{Z} G) \neq 0$. 
Proof. Let $C_{*}(X)$ denote a simplicial chain complex for $X$ and let $P_{*}$ be a free resolution for $\mathbb{Z}$ over $\mathbb{Z} G$. Consider the spectral sequence for

$$
E_{0}^{i j}=\operatorname{Hom}_{G}\left(C_{i}(X) \otimes P_{j}, M\right),
$$

where $M \cong \bigoplus_{k} \mathbb{Z} G$ (compare ChapterVII.7 in [Bro1]). For each fixed $i, C_{i}(X) \otimes P_{*}$ is a free resolution of $C_{i}(x)$, so in the spectral sequence in which the differential $d_{0}: E_{0}^{i j} \rightarrow E_{0}^{i, j+1}$ is induced by the differential on $P_{*}$, we have $E_{1}^{i j} \cong \operatorname{Ext}_{G}^{j}\left(C_{i}, M\right)$. By Shapiro's lemma (cf., e.g., Proposition III.6.2 in [Bro1]), $\operatorname{Ext}_{G}^{*}(\mathbb{Z} G / H, M) \cong$ $\operatorname{Ext}_{H}^{*}(\mathbb{Z}, M)=H^{*}(H ; M)$ for a subgroup $H<G$. By the hypotheses, for a stabilizer $H$ of every simplex in $X$, we have $H^{j}(H ; M)=0$ for $j>0$. Hence $\operatorname{Ext}_{G}^{j}\left(C_{i}, M\right)=0$ for $j>0$ and we have $E_{1}^{i j}=0$ for $j>0$, and $E_{1}^{i 0}=$ $\operatorname{Hom}_{G}\left(C_{i}, M\right)$. Thus the spectral sequence collapses at $E_{2}$, with $E_{2}^{i j}=0$ for $i>0$ and $E_{2}^{i 0}=H_{G}^{i}(X ; M)$. On the other hand, the total complex for $C_{*} \oplus P_{*}$ is a free resolution for $\mathbb{Z}$ over $\mathbb{Z} G$, and so the spectral sequence must converge to a filtration of $H^{*}(G ; M)$. Thus we have a natural isomorphism between $H^{*} \operatorname{Hom}_{G}\left(C_{*}(X) ; M\right)$ and $H^{*}(G ; M)$ (compare Exercise VIII.7.4 in [Bro1]).

Since $C_{*}(X)$ is a chain complex of finitely generated $G$-modules we have

$$
\operatorname{Hom}_{G}\left(C_{*}(X), \bigoplus_{j} \mathbb{Z} G\right)=\bigoplus_{j} \operatorname{Hom}_{G}\left(C_{*}(X), \mathbb{Z} G\right) .
$$

Now taking cohomology we obtain

$$
\begin{aligned}
H^{*}(G ; M) & =H^{*} \operatorname{Hom}_{G}\left(C_{*}(X) ; M\right) \\
& =\bigoplus_{k} H^{*} \operatorname{Hom}_{G}\left(C_{*}(X) ; \mathbb{Z} G\right) \\
& =\bigoplus_{k} H^{*}(G ; \mathbb{Z} G) .
\end{aligned}
$$

Using Proposition 2.8 and setting $M=\widetilde{F}$ we have that there exists $n$ such that $H^{n}(G ; \mathbb{Z} G) \neq 0$.

2.3. Connectedness and acyclicity at infinity. For a topological space $Y$ and an integer $n \geq-1$ denote by $\operatorname{Conn}_{n}^{\infty}(Y)$ the following condition:

For every compact $K \subset Y$ there exists a compact $L \subset Y$ such that $K \subset L$ and every map $S^{n}=\partial B^{n+1} \rightarrow Y \backslash L$ extends to a map $B^{n+1} \rightarrow Y \backslash K$. This condition is also called vanishing of the $n$-th homotopy pro-group at infinity - see [G].

If $\operatorname{Conn}_{k}^{\infty}(Y)$ holds for every $k \leq n$ then we say that $Y$ is $n$-connected at infinity (compare e.g. [G]). $Y$ is (-1)-connected at infinity if and only if it is not compact. $Y$ is 0-connected at infinity if and only if it has one end. A space 1-connected at infinity is also called simply connected at infinity. 
Recall that two maps $f_{1}, f_{2}: X \rightarrow Y$ are said to be properly homotopic if there exists a proper (i.e. with compact preimages of compact sets) homotopy $F: X \times[0,1] \rightarrow Y$ joining them. A proper map $f: X \rightarrow Y$ is a proper homotopy equivalence if there exists a proper map $g: Y \rightarrow X$ such that $f \circ g$ and $g \circ f$ are properly homotopic to, respectively $\mathrm{id}_{Y}$ and $\mathrm{id}_{X}$. It is obvious that if $f: X \rightarrow Y$ is a proper homotopy equivalence then $\operatorname{Conn}_{n}^{\infty}(X)$ if and only if $\operatorname{Conn}_{n}^{\infty}(Y)$.

A map $f: X \rightarrow Y$ between CW complexes is $C W$-proper if for each $n$ there exists $k$ such that $f\left(X^{(n)}\right) \subset Y^{(k)}$ and $\left.f\right|_{X^{(n)}}: X^{(n)} \rightarrow Y$ is proper. A definition of $C W$-proper homotopy equivalence is then analogous to proper homotopy equivalence.

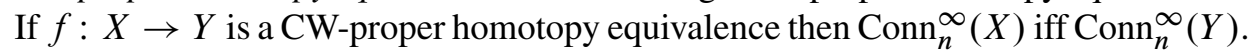

The next theorem is a slight generalization of a part of Theorem 5.8.2 of [G]. The proof is the same as the one of the latter (this was pointed out to us by Ross Geoghegan) hence we just describe it briefly. An action of a group on a CW complex is rigid if the stabilizer of any cell acts trivially on that cell. Observe that a group acting by simplicial automorphisms on a simplicial complex acts rigidly on the first barycentric subdivision of the complex. Let $\pi: A \rightarrow C$ be a cellular map between $\mathrm{CW}$ complexes and let for each cell $e$ of $C$ a CW complex $F_{e}$ be given. Then (see e.g. Chapter 2.6 in [G]) $\pi$ is a stack of $C W$ complexes with fiber $F_{e}$ if it is (roughly speaking) "built" by induction on skeleta of $C$ so that over the interior $\stackrel{\circ}{e}$ of a cell $e$, $\pi$ is homotopically equivalent to the projection $F_{e} \times \stackrel{\circ}{\rightarrow} \rightarrow \stackrel{\circ}{e}$.

Proposition 2.10. Let $Y_{1}$ and $Y_{2}$ be two contractible rigid $G-C W$ complexes with cocompact $G$-actions such that stabilizers of all cells are finite. Then $\operatorname{Conn}_{n}^{\infty}\left(Y_{1}\right)$ if and only if $\operatorname{Conn}_{n}^{\infty}\left(Y_{2}\right)$.

Proof. For $i=1,2$ apply the Borel Construction (see e.g. Chapter 2.6 in [G]) using a $K(G, 1)$-complex $X$ of finite type to get the commutative diagram:

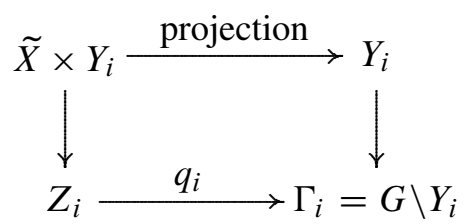

Here, the diagonal action of $G$ on the contractible $\mathrm{CW}$ complex $\tilde{X} \times Y_{i}$ is free, so $Z_{i}=G \backslash\left(\tilde{X} \times Y_{i}\right)$ is a $K(G, 1)$-complex. Then $q_{i}: Z_{i} \rightarrow \Gamma_{i}$ is a stack of $\mathrm{CW}$ complexes which can be rebuilt to give a commutative diagram

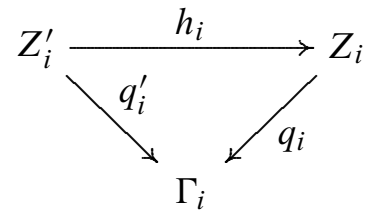


(see 2.6.4 in [G]) in which $q_{i}^{\prime}: Z_{i}^{\prime} \rightarrow \Gamma_{i}$ is a stack of CW complexes and $h_{i}$ is a homotopy equivalence. Here the fiber $F_{e}$ of $q_{i}$ over the cell $e$ of $\Gamma_{i}$ is a $K\left(G_{e}, 1\right)$ complex where $G_{e}$ is finite, and the fiber $F_{e}^{\prime}$ of $q_{i}^{\prime}$ over $e$ is a $K\left(G_{e}, 1\right)$-complex of finite type (see 2.11.5 of [G]). From Chapters 2.6 and 2.12 of [G] one sees that the map $\widetilde{Z}_{i}^{\prime} \rightarrow \tilde{X} \times Y_{i} \rightarrow Y_{i}$ is a stack of CW complexes in which the fiber over a cell $\tilde{e}$ of $Y_{i}$ is homeomorphic to the universal cover of $F_{e}^{\prime}$ (where $\tilde{e}$ lies over a cell $e$ of $\Gamma_{i}$ ). Thus that fiber is a contractible $\mathrm{CW}$ complex of finite type. It follows that the indicated map $\widetilde{Z}_{i}^{\prime} \rightarrow Y_{i}$ is a CW-proper homotopy equivalence and hence $\operatorname{Conn}_{n}^{\infty}\left(Y_{i}\right)$ if and only if $\operatorname{Conn}_{n}^{\infty}\left(\widetilde{Z}_{i}^{\prime}\right)$.

Thus to prove the proposition it suffices to show that $\operatorname{Conn}_{n}^{\infty}\left(\widetilde{Z}_{1}^{\prime}\right)$ is equivalent to $\operatorname{Conn}_{n}^{\infty}\left(\widetilde{Z}_{2}^{\prime}\right)$. But $Z_{1}^{\prime}$ and $Z_{2}^{\prime}$ are both $K(G, 1)$-complexes hence by Corollary 2.8.7 of [G] they are homotopy equivalent and by Theorem 3.1.23 of [G] there exists a proper homotopy equivalence $\widetilde{Z}_{1}^{\prime} \rightarrow \widetilde{Z}_{2}^{\prime}$.

Hence for a group $G$ we can define a condition $\operatorname{Conn}_{k}^{\infty}(G)$ by requiring that $\operatorname{Conn}_{k}^{\infty}(X)$ holds for some, and hence for any contractible, rigid $G$-CW complex $X$ on which $G$ acts properly discontinuously and cocompactly. If $\operatorname{Conn}_{k}^{\infty}(G)$ for every $k=-1,0,1, \ldots, n$ then we say that $G$ is $n$-connected at infinity (cf [G] Chapter 5.4).

Note that, by Theorems 7.6.11 and 7.6.12 of [G], $\operatorname{Conn}_{k}^{\infty}(G)$ is a quasi-isometry invariant in the sense that if $G$ and $H$ are quasi-isometric groups then $\operatorname{Conn}_{k}^{\infty}(G)$ if and only if $\operatorname{Conn}_{k}^{\infty}(H)$.

Let $Y$ be a finite-dimensional locally finite path connected CW complex, let $\left(L_{i}\right)_{i=1}^{\infty}$ be the filtration of $Y$ by compact subcomplexes and let $n \geq-1$ be an integer. We denote by $Y-{ }_{c} L_{i}$ the largest subcomplex of $Y$ whose vertices are the vertices of $Y \backslash L_{i}$. We say that $Y$ is $n$-acyclic at infinity with respect to a $\operatorname{ring} R$ if the inverse system $\left\{\tilde{H}_{k}\left(Y-{ }_{c} L_{i} ; R\right), l_{i}^{j}\right\}$ is pro-trivial for every $-1 \leq k \leq n$ (see e.g. Chapter 5.8 in [G]). Here $l_{i}^{j}: H_{k}\left(Y-{ }_{c} L_{j} ; R\right) \rightarrow H_{k}\left(Y{ }_{-c} L_{i} ; R\right)$ is a map between reduced homologies induced by inclusion. An inverse system like the above is pro-trivial if for every $i$ there exists $j \geq i$ such that the image of $l_{i}^{j}$ is trivial.

Let moreover $Y$ be a contractible rigid $G$-CW complex with cocompact $G$-action such that stabilizers of all cells are finite. If $Y$ is $n$-acyclic at infinity then we say that the group $G$ is $n$-acyclic at infinity. By Theorem 5.8.2 in [G] this is a property of $G$, i.e. it does not depend on the choice of $Y$ as above.

2.4. Semistability at infinity. Let $G$ act geometrically (i.e. properly discontinuously and cocompactly) and rigidly on a one ended contractible CW complex $Y$. Let $\left(L_{i}\right)_{i=1}^{\infty}$ be the filtration of $Y$ by compact subcomplexes. Choose a map $\omega:[0,+\infty) \rightarrow Y$ such that $\omega(i) \in Y-{ }_{c} L_{i}, i \in \mathbb{N}$, and put $\pi_{1}^{i}=\pi_{1}\left(Y-{ }_{c} L_{i}, \omega(i)\right)$ and denote by $P_{i}^{j}: \pi_{1}^{j} \rightarrow \pi_{1}^{i}$ the maps induced by inclusions and changing of the base point along $\omega$. Then we say (see [G], Chapters 5.1, 5.4 and Theorem 5.8.2) that a group $G$ is semistable at infinity if for every $i$ there exists $j \geq i$ such that for all $k \geq j$ we have 
$\operatorname{im}\left(P_{i}^{j}\right)=\operatorname{im}\left(P_{i}^{k}\right)$. Note that this definition does not depend on the choice of the filtration $\left(L_{i}\right)_{i=1}^{\infty}$.

If $G$ is semistable at infinity then the fundamental pro-group at infinity of $G$ being the inverse limit of the system $\left\{\pi_{1}^{i}, P_{i}^{j}\right\}$ is well defined, i.e., it is independent of $Y$ and $\omega$. Moreover in that case some other invariants (e.g. Čech fundamental group or strong fundamental group) can be defined (see Section 5.4 of [G]).

It is still unknown if there exists a finitely presented one-ended group which is not semistable at infinity.

\section{Connectedness at infinity of systolic complexes and groups}

In this section we show that for a systolic group $G$ and a natural $k \geq 2$ the condition $\operatorname{Conn}_{k}^{\infty}(G)$ holds (see section 2.3 for the definition). As a consequence we get that systolic groups are not simply connected at infinity. Those results allow us to distinguish systolic groups from some other natural classes of groups.

Theorem 3.1. Let $X$ be a locally finite systolic complex, let $G$ be a systolic group and let $k \geq 2$ be a natural number. Then the conditions $\operatorname{Conn}_{k}^{\infty}(X)$ and $\operatorname{Conn}_{k}^{\infty}(G)$ hold.

Proof. Let a compact $K \subset X$ be given. Choose a simplex $\tau$ of $X$. We denote by $B_{l}$ the combinatorial ball $B_{l}(\tau, X)$.

There exists a natural $l>0$ such that $K$ is a subset of $B_{l}$. Define $L=B_{l+2}$. Let $M=X-{ }_{c} B_{l+1}$ be the maximal subcomplex of $X$, whose vertices are the vertices of $X \backslash B_{l+1}$. By definition $M$ is a full subcomplex of $X$ and hence, by Fact 2.1, it is aspherical. Therefore every map $f: S^{k} \rightarrow X \backslash L \subset M$ extends to a map $g: B^{k+1} \rightarrow M \subset X \backslash K$. Thus $\operatorname{Conn}_{k}^{\infty}(X)$ holds.

To show that $\operatorname{Conn}_{k}^{\infty}(G)$ holds, it is enough to notice that if $G$ acts properly discontinuously and cocompactly by automorphisms on a systolic complex $Y$ then $G$ acts geometrically and rigidly on the first barycentric subdivision $Y^{\prime}$ of $Y$. Thus $\operatorname{Conn}_{k}^{\infty}(Y)$ implies $\operatorname{Conn}_{k}^{\infty}\left(Y^{\prime}\right)$ and the latter is equivalent to $\operatorname{Conn}_{k}^{\infty}(G)$.

Theorem 3.2. Systolic groups are not simply connected at infinity.

Proof. Let $G$ be a systolic group and let $X$ be a systolic simplicial complex on which $G$ acts geometrically, by automorphisms.

We will prove that $G$ is not simply connected at infinity, arguing by contradiction. Assume $G$ is simply connected at infinity. Then condition $\operatorname{Conn}_{k}^{\infty}(G)$ holds for every $k=-1,0,1,2, \ldots$, i.e., $G$ is $l$-connected at infinity for arbitrarily large $l$. By the Proper Hurewicz Theorem (Theorem 5.7.6 in [G]) we have that $G$ is $l$-acyclic at infinity with respect to $\mathbb{Z}$ for arbitrarily large $l$. By Corollary 4.2 in [GMi1] (compare 
also Theorem in [GMi2] and Theorem 4.3.3 in [G]) it follows that $H^{k}(G ; \mathbb{Z} G)=0$ for all $k$.

This is a contradiction since, by Proposition 2.9, there exists a natural number $n \leq \operatorname{dim}(X)<\infty$ such that $H^{n}(G ; \mathbb{Z} G) \neq 0$.

Corollary 3.3. Let $0 \rightarrow K \rightarrow G \rightarrow H \rightarrow 0$ be a short exact sequence of infinite finitely presented groups. If $G$ is systolic then neither $K$ nor $H$ has one end.

Proof. It follows directly from Corollary 1.5 in [GMi1] (compare also Theorem 5.9.7 in $[\mathrm{G}]$ ) and the fact that $G$ is not simply connected at infinity.

3.1. Non-examples. Here we list some corollaries of the above results.

1) $\mathbb{Z}^{3}$ is not systolic (see below). This is also proved in [W] by showing that the second isoperimetric function of a systolic group is linear (for a precise definition and a proof see Section 8 of [JS2]). Note that it is conjectured there that higher isoperimetric functions are also linear.

2) Let $M$ be a closed manifold. Then $M$ has homotopy type of a finite polyhedron $P$ (cf. $[\mathrm{KS}]$ ). The universal covers $\tilde{M}$ and $\widetilde{P}$ of, respectively $M$ and $P$ are properly homotopy equivalent. Hence if $\tilde{M}=\mathbb{R}^{n}$ for $n \geq 3$, then $\widetilde{P}$ is simply connected at infinity. Thus we get the following.

Corollary 3.4. For $n \geq 3$, fundamental groups of closed manifolds covered by $\mathbb{R}^{n}$ are not systolic.

3) A theorem of D. Wise on subgroups (see [W]) says that finitely presented subgroups of a fundamental group of a compact 6-large complex are systolic. Hence we get that fundamental groups of closed manifolds covered by $\mathbb{R}^{n}, n \geq 3$, are not isomorphic to subgroups of fundamental groups of compact 6-large complexes.

In [JS2] it is proved directly (i.e. without using the result of Wise) that fundamental groups of closed non-positively curved riemannian manifolds of dimension at least three are not isomorphic to subgroups of systolic groups.

4) From Corollary 3.3 it follows that if a systolic group $G$ is a product $G=H \times K$ of infinite groups, then neither $K$ nor $H$ is one-ended. To show this observe that $G$ is finitely presented and therefore its factors $H$ and $K$ are finitely presented, too.

Corollary 7.5 in [JS2] states that in fact a product of infinite groups $H$ and $K$ is systolic only if $H$ and $K$ are both virtually free.

5) By Corollary 1.5 in [GMi1] (compare Corollary 5.9.6 in [G]) a product of infinite finitely generated groups has one end. Hence, analogously to point 4) above, we have that if $G=H \times K \times N$, where $H, K$ and $N$ are infinite then $G$ is not a systolic group.

This gives another proof of Corollary 7.7 in [JS2]. 
6) Coxeter groups acting cocompactly on $\mathbb{R}^{n}$ for $n \geq 3$ are not systolic. Moreover, let $(W, S)$ be a Coxeter system, $L$ the associated nerve and $\delta$ the poset of spherical subsets of $S$ (we use the notation of [DM]). Assume $L-\sigma$ is simply connected for every $\sigma \in \mathcal{~}$. Then by Theorem 4.3 in [DM] $W$ is simply connected at infinity and $W$ is not systolic. (Compare point 8 ) below).

7) Let $X$ be a locally compact building (triangulated) with an apartment isometric to $A$ (for basic facts about buildings see eg. [Bro2]). Let $A$ be a space for which $\operatorname{Conn}_{k}^{\infty}(A)$ does not hold for some $k \geq 2$. Then $\operatorname{Conn}_{k}^{\infty}(X)$ does not hold. Hence discrete and cocompact subgroups of automorphisms group of $X$ are not systolic.

To show this let us fix a folding $p: X \rightarrow A$ onto an apartment of $X$ such that $p^{-1}(b)=b$ for some chamber $b$. Take a natural $k \geq 2$ and a compact set $\bar{K} \subset A$ such that for every compact $\bar{L} \supset \bar{K}$ there exists a map $f: S^{k} \rightarrow A \backslash \bar{L}$ non homotopically trivial in $A \backslash \bar{K}$. Let $K=p^{-1}(\bar{K})$ and let a compact $L \subset X$ with $K \subset L$ be given (here we use local compactness of $X$ ). Then $\bar{L}=p(L)$ is compact and one can find a map $f$ as above. To show that $f$ is homotopically nontrivial in $X \backslash K$ we will argue by a contradiction. Let us assume there exists its extension $g: B^{k+1} \rightarrow X \backslash K$. Then $p \circ g: B^{k+1} \rightarrow A \backslash \bar{K}$ is an extension of $f: S^{k} \rightarrow A \backslash \bar{L}$ that contradict non triviality of $f$ in $A \backslash \bar{K}$. In particular cocompact lattices in groups of automorphisms of Euclidean (i.e. with apartments being the Euclidean spaces $\mathbb{E}^{n}$ ) and hyperbolic (with apartments $\mathbb{H}^{n}$ ) buildings are not systolic for $n \geq 3$.

8 ) Let $X$ be a finite, locally CAT(0), piecewise Euclidean complex. For a cell $\sigma$ of $X$ let $\operatorname{Lk}(\sigma)$ denote its link, and for $p \in \operatorname{Lk}(\sigma)$ let $\operatorname{Plk}(\sigma, p)$ denote the punctured link of $\sigma$ at $p$, i.e., $\operatorname{Lk}(\sigma)$ with all the points closer than $\pi / 2$ to $p$ (in the angular metric on $\operatorname{Lk}(\sigma)$ ) removed (we use here the notation of [BMcCM]). Assume that for every cell $\sigma$ of $X$ and every point $p \in \operatorname{Lk}(\sigma)$ both $\operatorname{Lk}(\sigma)$ and $\operatorname{Plk}(\sigma, p)$ are simply connected. Then, by Theorem 1.3 in [BMcCM], the universal cover $\tilde{X}$ of $X$ is simply connected and hence the fundamental group of $X$ is not systolic.

Questions. Do there exist closed aspherical manifolds of dimension above 2, whose fundamental groups are systolic?

More generally, does there exist a closed aspherical manifold $M$ of dimension $n \geq 3$, such that $\operatorname{Conn}_{k}^{\infty}(\tilde{M})$ holds for every $k \geq 2$ ?

For Davis aspherical manifolds $M$ (cf. [D]), $\operatorname{Conn}_{k}^{\infty}(M)$ fails for some $k \geq 2$ (this fact we know from Craig Guilbault and Tadeusz Januszkiewicz).

\section{Systolic chamber complexes}

The aim of this section is to show that systolic complexes of some class are oneended, are not simply connected at infinity, and groups acting on them geometrically are semistable at infinity. That class contains, in particular, systolic groups acting on normal pseudomanifolds of arbitrarily large dimension, constructed in [JS1]. 
Throughout this section, if it is not stated otherwise, we denote by $X$ a systolic chamber complex of dimension $n \geq 1$. For a natural $k$, we denote by $B_{k}$ the closed combinatorial ball $B_{k}(\tau, X)$ around a given simplex $\tau$ of $X$.

Lemma 4.1. $S_{k}$ is an (n-1)-dimensional chamber complex for every $k \geq 1$.

Proof. We first show that $S_{k}$ is at most $(n-1)$-dimensional. If there existed an $l$-simplex $\sigma$ in $S_{k}$ with $l \geq n$, then one would have a projection of $\sigma$ onto $B_{k-1}$ (see Lemma 2.3) spanning with $\sigma$ a simplex of dimension $>n$. Now we show that every $l$-simplex $\rho$ of $S_{k}$ is contained in some $(n-1)$-simplex by induction on the dimension of $X$. For $\operatorname{dim}(X)=1$ the assertion is clear. Let now $\operatorname{dim}(X)=n>1$. Consider the link $X_{\rho}$. It is obviously an $(n-l-1)$-dimensional chamber complex. Moreover (see Lemma 2.3) $X_{\rho} \cap B_{k}$ is a ball $B^{\prime}$ in $X_{\rho}$ of radius 1 around some simplex $v$ of $X_{\rho}$. But $X_{\rho}$ is a 6-large complex and closed balls of radii less than 3 in such complexes are the same as those in their universal covers, i.e. as in systolic complexes (see Lemma 2.5). Hence by the induction assumptions there exists $(n-l-2)$-simplex $\kappa$ in $S^{\prime}=\partial\left(X_{\rho} \cap B_{k}\right)=X_{\rho} \cap S_{k}$ (see Lemma 2.3). Then $\rho * \kappa$ is an $(n-1)$-simplex of $S_{k}$.

Now we show that every codimension one simplex of $S_{k}$ is contained in at least two maximal simplices of $S_{k}$. If $\rho$ (as above) is an $(n-2)$-simplex then $X_{\rho}$ is a 1-dimensional chamber complex and $X_{\rho} \cap B_{k}=B_{1}\left(v, X_{\rho}\right)$. Since $X_{\rho}$ is a chamber complex, there should be at least two edges in $X_{\rho} \cap B_{k}$ intersecting $v$ with other vertices $\kappa_{1}$ and $\kappa_{2}$ lying on $S_{1}\left(v, X_{\rho}\right)$. Then $\rho * \kappa_{1}$ and $\rho * \kappa_{2}$ are two $(n-1)$ simplices containing $\rho$.

Lemma 4.2. Let $\sigma$ be an $(n-1)$-dimensional simplex of $S_{k}$. Then there exists a vertex $v$ at a distance $k+1$ from $\tau$ such that $v * \sigma$ is a simplex of $X$.

Proof. Since $\sigma$ belongs to $S_{k}$, there exists a vertex $w$ at distance $k-1$ from $\tau$ such that $w * \sigma$ is a simplex. Since $X$ is a chamber complex, there is another vertex $v$ spanning an n-simplex with $\sigma$. By Lemma 4.1 the vertex $v$ does not belong to $S_{k}$. Thus it remains to show that $v$ is not at a distance $k-1$ from $\tau$. Suppose it is. Then $P_{B_{k-1}}\left(b_{\sigma}\right)$ is, by definition, a barycenter of some simplex $\rho$ containing both $v$ and $w$. But then one has a simplex $\rho * \sigma$ belonging to $X$ and of dimension at least $n+1$. This contradicts the assumption on the dimension of $X$.

Corollary 4.3. The universal cover of a 6-large chamber complex of dimension at least 1 is unbounded.

Proof. By Lemmas 4.1 and 4.2 spheres of arbitrarily large radii around some simplex of the universal cover (which is systolic) are non-empty. 
Let us define here, for a given vertex $v$ of $X$, a condition $R(v, X)$ that will be crucial for the remaining part of the section:

$$
R(v, X) \text { if and only if } \quad\left(X_{v} \backslash \stackrel{\circ}{B}_{2}\left(\sigma, X_{v}\right) \text { is connected for all } \sigma \in X_{v}\right. \text {. }
$$

Lemma 4.4. Suppose that condition $R(v, X)$ holds for every vertex $v$ in $S_{k}$. Then for every path $\gamma_{k}=\left(v_{1}, v_{2}, \ldots, v_{l}\right)$ in $\left(S_{k}\right)^{(1)}$ (i.e., the $v_{i}$ 's are consecutive vertices) there exists a path

$$
\gamma_{k+1}=\left(w_{1}^{1}, w_{1}^{2}, \ldots, w_{1}^{m(1)}, z_{1}, w_{2}^{1}, \ldots, z_{l-1}, w_{l}^{1}, w_{l}^{2}, \ldots, w_{l}^{m(l)}\right)
$$

in $S_{k+1}^{(1)}$ such that:

- $P_{B_{k}}\left(w_{i}^{j}\right)$ lies in the same simplex of $S_{k}$ as $v_{i}$, for $j=1,2, \ldots, m(i)$, and

- $P_{B_{k}}\left(z_{i}\right)$ lies in the same simplex of $S_{k}$ as the barycenter $e_{i}$ of the edge $\left(v_{i}, v_{i+1}\right)$.

Proof. By Lemma 4.1, for every $i=1,2, \ldots, l-1$ there exists an $(n-1)$-simplex $\rho_{i}$ containing $\left(v_{i}, v_{i+1}\right)$. By Lemma 4.2 we can find vertices $z_{i}$ in $S_{k+1}$ such that $z_{i} * \rho_{i}$ span a simplex in $X$. By the definition of $P_{B_{k}}$ we have that $P_{B_{k}}\left(z_{i}\right)$ lies at a distance not greater than 1 from $e_{i}$.

Observe that $z_{i}$ belongs to $\left(X_{v_{i}} \backslash \stackrel{\circ}{B}_{2}\left(\sigma_{i}, X_{v_{i}}\right)\right) \cap\left(X_{v_{i+1}} \backslash \stackrel{\circ}{B}_{2}\left(\sigma_{i+1}, X_{v_{i+1}}\right)\right)$, where $\sigma_{j}=X_{v_{j}} \cap B_{k-1}(\tau, X)$. Hence, by $R\left(v_{i+1}, X\right)$, one can connect $z_{i-1}$ and $z_{i}$ by $\left(w_{i}^{1}, w_{i}^{2}, \ldots, w_{i}^{m(i)}\right)$. Since by definition $P_{B_{k}}\left(w_{i}^{j}\right) \in B_{1}\left(v_{i}, S_{k}\right)$ we conclude these projections are as desired.

Lemma 4.5. Let $S_{k}$ be connected and let condition $R(v, X)$ hold for every vertex $v \in\left(S_{k}\right)^{(0)}$. Then $S_{k+1}$ is connected.

Proof. Let $w, z \in\left(S_{k+1}\right)^{(0)}$ and let $\mu$ and $v$ be the two maximal simplices of $S_{k}$ containing, respectively, $P_{B_{k}}(w)$ and $P_{B_{k}}(z)$. Choose vertices $w^{\prime}$ of $\mu$ and $z^{\prime}$ of $v$ and a path $\gamma_{k}$ in $\left(S_{k}\right)^{(1)}$ joining $w^{\prime}$ and $z^{\prime}$. Then by the above lemma there exists a path $\gamma_{k+1}=\left(v_{1}, \ldots, v_{l}\right)$ in $\left(S_{k+1}\right)^{(1)}$ such that $v_{1} \in X_{w^{\prime}} \backslash \stackrel{\circ}{B_{2}}\left(\sigma_{w^{\prime}}, X_{w^{\prime}}\right)$ and $v_{l} \in X_{z^{\prime}} \backslash \stackrel{\circ}{B_{2}}\left(\sigma_{z^{\prime}}, X_{z^{\prime}}\right)$ for appropriate $\sigma_{w^{\prime}}$ and $\sigma_{z^{\prime}}$ (i.e. as in Lemma 2.3). Since also $w \in X_{w^{\prime}} \backslash \stackrel{\circ}{B}_{2}\left(\sigma_{w^{\prime}}, X_{w^{\prime}}\right)$ and $z \in X_{z^{\prime}} \backslash \stackrel{\circ}{B}_{2}\left(\sigma_{z^{\prime}}, X_{z^{\prime}}\right)$ by condition $R(v, X)$ we can extend $\gamma_{k+1}$ to a path connecting $w$ and $z$.

Theorem 4.6. Let $X$ be a locally finite systolic chamber complex of dimension greater than one. Suppose that $R(v, X)$ holds for almost every vertex $v \in X$ (i.e. for all but finitely many). Then $X$ is not simply connected at infinity. Moreover, if $G$ acts properly and cocompactly on $X$ then $G$ is semistable at infinity. 
Proof. We show $X$ is not simply connected at infinity, arguing by contradiction. Assume $X$ is simply connected at infinity. Let $\tau \in X^{(0)}$. Let $N \in \mathbb{N}$ be such that for every vertex $v \in X \backslash \stackrel{\circ}{B}_{N}$ condition $R(v, X)$ holds. By simple connectedness at infinity there exists a natural $L$ such that every loop in $X \backslash \stackrel{\circ}{B}_{N+L}$ is contractible in $X \backslash \stackrel{\circ}{B}_{N}$. Since $S_{N}$ is a full subcomplex of $X$, it is 6-large. It is also finite. But by Corollary 4.3 its universal cover (i.e. a systolic complex) is infinite, hence $S_{N}$ is not simply connected and we can find a closed path $\gamma_{N}$ in $\left(S_{N}\right)^{(1)}$ which is homotopically non-trivial in $S_{N}$. Observe that, by Lemma 4.4, we can find a closed path $\gamma_{N+1} \in\left(S_{N+1}\right)^{(1)}$ such that $P_{B_{N}}\left(\gamma_{N+1}\right)$ is homotopic to $\gamma_{N}$ in $S_{N}$. Since $P_{B_{N}}$ is a deformation retraction (see 2.4) we obtain that $\gamma_{N+1}$ is homotopic to $\gamma_{N}$ within $B_{N+1} \backslash \stackrel{\circ}{B}_{N}$. Continuing this process we can find a closed path $\gamma_{N+L}$ in $\left(S_{N+L}\right)^{(1)}$ such that it is homotopic to $\gamma_{N}$ within $B_{N+L} \backslash \stackrel{\circ}{B}_{N}$ and is homotopically trivial within $X \backslash \stackrel{\circ}{B}_{N+L}$ by the assumptions on $L$. But then $\gamma_{N}$ is homotopic in $S_{N}$ to $P_{B_{N}}\left(\gamma_{N+L}\right)$ which is homotopically trivial within $S_{N}$. This contradicts the choice of $\gamma_{N}$.

To see that $G$ is not simply connected at infinity observe that $G$ acts properly discontinuously, cocompactly and rigidly on the barycentric subdivision of $X$ (see Section 2.3).

For semistability take a filtration $\left(B_{k}\right)_{k=0}^{\infty}$ of $X$ (see Section 2.4) and a suitable ray $\omega:[0,+\infty) \rightarrow X$ such that $P_{B_{k}}(\omega(k))=\omega(k-1)$ (this can be done by taking a sequence $\left(x_{k}\right)_{k=0}^{\infty}$ of points such that $x_{k} \in S_{k}$, then considering their projections on spheres $S_{i}$ which are compact and getting the desired sequence by a diagonal argument taking accumulation points of projections on $S_{i}$ 's). Observe that $X^{\prime}-{ }_{c} B_{k}$ (we take a barycentric subdivision $X^{\prime}$ of $X$ to make action of $G$ rigid) is homotopically equivalent to $X \backslash \stackrel{\circ}{B}_{k}$ and hence to $S_{k+1}$. Given $k \geq N$ ( $N$ as above) and an element $g \in \pi_{1}\left(X-{ }_{c} B_{k}, \omega(k)\right)=\pi_{1}\left(S_{k+1}, \omega(k)\right)$, choose a path $\gamma$ in $\left(S_{k+1}\right)^{(1)}$ representing $g$. Then by the above construction for any $L=0,1,2, \ldots$ one can find a closed path $\gamma_{L}$ in $\left(S_{k+L+1}\right)^{(1)}$ such that $P_{B_{k+1}}\left(\gamma_{L}\right)$ is homotopic to $\gamma$ in $S_{k+1}$. Hence if $g_{L}$ is an element of $\pi_{1}\left(S_{k+L+1}, \omega(k+L)\right)=\pi_{1}\left(X-{ }_{c} B_{k+L}, \omega(k+L)\right)$ represented by $\gamma_{L}$ (using connectedness of $S_{k+L+1}$ by Lemma 4.5) we have that $\left(P_{B_{k+1}}\right)_{*}\left(g_{L}\right)=g$ and that the map $\pi_{1}\left(X-{ }_{c} B_{k+L}, \omega(k+L)\right) \rightarrow \pi_{1}\left(X-{ }_{c} B_{k}, \omega(k)\right)$ induced by the inclusion is surjective. This shows $G$ is semistable at infinity.

The next lemma gives a condition which helps to prove the condition $R(v, X)$ in some cases - see e.g. Section 5 .

Lemma 4.7. Let $X$ be a 6-large chamber complex such that $X_{\kappa}$ is connected for every simplex $\kappa$ of codimension greater than one in $X$ and $X_{\sigma} \backslash \stackrel{\circ}{B}_{2}\left(\rho, X_{\sigma}\right)$ is connected for every codimension two simplex $\sigma$ of $X$ and every simplex $\rho$ of its link $X_{\sigma}$. Then $R(v, X)$ holds for every vertex $v$ of $X$.

Proof. We will proceed by induction on $n=\operatorname{dim}(X)$. 
For $n=2$ the assertion is clear since codimension two simplices are just vertices. Assume we proved the lemma for $n \leq k$. Let $\operatorname{dim}(X)=k+1$. Take a vertex $v$ of $X$ and consider its link $X_{v}$. It has dimension $k$. Observe that if $\alpha$ is a codimension $l$ simplex of $X_{v}$ then $\beta=\alpha * v$ is a codimension $l$ simplex of $X$ and $\left(X_{v}\right)_{\alpha}=X_{\beta}$. Hence by the induction assumptions for every vertex $w$ of $X_{v}$ condition $R(w, X)$ holds.

Take a simplex $\omega$ of $X_{v}$. Since $\omega=S_{0}\left(\omega, X_{v}\right)$ is connected we have by Lemma 4.5 , Lemma 2.5 and by the fact that $X_{v}$ is 6-large (as a full subcomplex of $X)$ that also $S_{1}\left(\omega, X_{v}\right)$ and $S_{2}\left(\omega, X_{v}\right)$ are connected. Take two vertices $t$ and $s$ of $X_{v} \backslash \stackrel{\circ}{B}_{2}\left(\omega, X_{v}\right)$. Since, by assumptions, $X_{v}$ is connected there exists a path in $\left(X_{v}\right)^{(1)}$ joining them. If this path misses $S_{2}\left(\omega, X_{v}\right)$ it joins these vertices in $X_{v} \backslash \stackrel{\circ}{B_{2}}\left(\omega, X_{v}\right)$. If not, we can replace it (using connectedness of $S_{2}\left(\omega, X_{v}\right)$ ) by a path in $X_{v} \backslash \stackrel{\circ}{B_{2}}\left(\omega, X_{v}\right)$.

Hence we have the conclusion.

Corollary 4.8. Let $X$ be locally finite systolic chamber complex of dimension above one. If $X_{\kappa}$ is connected for every simplex $\kappa$ of codimension greater than one in $X$ and $X_{\sigma} \backslash \stackrel{\circ}{B}_{2}\left(\tau, X_{\sigma}\right)$ is connected for every codimension two simplex $\sigma$ of $X$ and every simplex $\tau$ of its link $X_{\sigma}$, then $X$ is not simply connected at infinity and every group acting properly and cocompactly on $X$ is semistable at infinity.

Remark. Observe that it follows from the proof of Lemma 4.7 that it is enough to have the above assumptions only for almost every codimension two simplex $\sigma$ similarly as in Theorem 4.6.

Corollary 4.9. Locally finite normal systolic pseudomanifolds are one-ended and are not simply connected at infinity. Groups acting on them cocompactly and properly are one-ended, semistable at infinity and do not split as amalgamated products or as HNN extensions over finite groups.

Proof. Let $X$ be a locally finite normal systolic pseudomanifold. Let $\sigma$ be an $l$ simplex of $X$, for $l \geq 2$. Then $\partial \sigma$ is connected and by Lemma 4.5 every sphere $S_{i}(\sigma, X)$ is connected. Thus $X$ has one end.

As for semistability at infinity observe that one dimensional link of a normal pseudomanifold is a circle. Hence it satisfies the assumptions of the preceding corollary.

By Stallings theorem ([S]) if a finitely generated group has one end then it does not split as an amalgamated product or an HNN extension over a finite group.

Remark. Systolic groups acting geometrically on normal pseudomanifolds constructed in [JS1] are the only systolic groups of cohomological dimension greater then two known to us at the moment. 
Example. We give an example of a 2-dimensional systolic normal chamber complex $X$ which is simply connected at infinity. It shows we cannot delete condition $R(v, X)$ from the assumptions of Theorem 4.6. Let $(A, a, b, r)$ denote the quadruple consisting of

- $A$, a closed Euclidean half-plane (i.e. $\left\{(x, y) \in \mathbb{R}^{2} \mid y \geq 0\right\}$ ) triangulated by regular triangles;

$-a$, a vertex (of triangulation) in the interior of $A$;

$-b$, a vertex on the boundary $\partial A$;

- $r$, a closed geodesic ray in $A^{(1)}$ starting from $a$.

Let $\left(A_{i}, a_{i}, b_{i}, r_{i}\right)_{i=1}^{\infty}$ be a sequence of quadruples isomorphic to $(A, a, b, r)$. We will construct $X$ recursively by "gluing consecutive quadruples". More precisely let $X_{1}=A_{1}$. Having $X_{k}$ we find the lowest index $i_{k} \leq k$ such that $b_{i_{k}}$ belongs to a boundary edge $e_{k}$ of $A_{i_{k}}$ (viewed as a subspace of $X_{k}$ ) that does not belong to any triangles of $A_{j}$ (viewed as a subspace of $X_{k}$ ), for $j \neq i_{k}, j \leq k$. Then $X_{k+1}=X_{k} \cup_{\psi_{k}} A_{k+1}$, where $\psi_{k}: r_{k+1} \rightarrow A_{i_{k}}$ sends isometrically $r_{k+1}$ to a closed half-line $\bar{r}_{k+1}$ of $\partial A_{i_{k}}$ starting at $b_{i_{k}}$ and containing $e_{k}$. If one defines a map $\phi_{k}: X_{k+1} \rightarrow X_{k}$ so that $\phi_{k}\left(A_{k+1}\right)=\bar{r}_{k+1}$ and as identity on $X_{k} \subset X_{k+1}$, then $\left(X_{i}, \phi_{i}\right)_{i=1}^{\infty}$ is an inverse sequence and we set $X=\operatorname{inv} \lim \left(X_{i}, \phi_{i}\right)$.

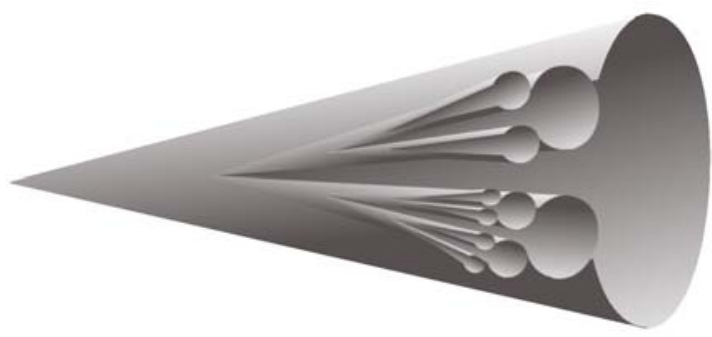

Figure 1. Topology of $X_{11}$.

It is clear that $X$ is a locally finite 2- dimensional normal systolic chamber complex. Since every $A_{i}$ is contractible and we "glue them along rays" we obtain that $X$ is contractible. Then it can be easily observed that $X$ is simply connected at infinity. Observe that the assumptions of Theorem 4.6 (i.e. the condition $R\left(b_{i}, X\right)$ ) are not satisfied for vertices $b_{i}$ in $X$. Note that there is no geometric group action on $X$.

Question. Are one-ended systolic groups semistable at infinity? 


\section{Extra-tileable simplices of groups}

Besides groups acting geometrically on trees and on 2-dimensional systolic complexes the only other systolic groups known to us at the moment are the ones constructed in [JS1] as fundamental groups of locally 6-large and locally extra-tilable simplices of groups (see the comment after Proposition 2.7 in Section 2).

In this section we show the latter groups are one-ended and semistable at infinity, provided groups associated with codimension one faces are nontrivial. Let $\mathcal{G}$ be a locally 6-large and locally extra-tilable simplex of groups, as constructed in Proposition 19.1 of [JS1] and let $\Delta$ be the underlying simplex. Assume that groups associated with codimension one faces of $\Delta$ are nontrivial. In view of Corollary 4.8 it is enough to show that the universal development $\widetilde{\mathscr{G}}$ of $\mathscr{G}$ (which exists by Proposition 2.6) is a locally finite systolic chamber complex such that its links of dimension above 0 are connected and complements of open balls of radius 2 in 1-dimensional links of $\widetilde{\mathscr{g}}$ are connected. The argument below was supplied by J. Świątkowski.

We refer to the construction made in [JS1], Section 19, and use notation introduced there. See also Section 2 above.

By Proposition 17.1 (5) of [JS1] (compare also [BriH]) the universal development $\widetilde{\mathscr{E}}$ is a union of its top-dimensional simplices and, by our assumption, the groups $G_{\tilde{\sigma}}$ (compare Section 2) are non-trivial for all codimension one faces $\sigma$ of $\Delta$. Hence $\widetilde{\mathscr{E}}$ is a chamber complex. By Proposition 19.1 of [JS1] it is locally 6-large and hence because it is simply connected - systolic. It is locally finite by Proposition 19.1 (3) of [JS1], because the groups $G_{\sigma}$ are finite for all faces $\sigma$ of $\Delta$ (by the proof of Proposition 19.1 of [JS1]). By construction (the proof of Proposition 19.1 of [JS1]), for every face $\sigma$ of $\Delta$ the canonical morphism $i_{\sigma}: \mathscr{E}^{\sigma} \rightarrow G_{\sigma}$ is surjective (meaning that $G_{\sigma}$ is generated by the union of images of $i_{\sigma}$ 's). Hence, by Proposition 17.1 (6) of [JS1], links in $\widetilde{\mathscr{g}}$ are gallery connected.

Now we show that complements of open balls of radius 2 in 1-dimensional links of $\widetilde{\mathscr{E}}$ are connected. Let $\sigma$ be a codimension 2 face of $\Delta$ and $s, t$ be the two codimension 1 faces containing $\sigma$. Then the groups $G_{s}, G_{t}$ and $G_{\Delta}$ form a segment of groups $\mathscr{E}^{\sigma}$ over the link $\Delta_{\sigma}$, which is extra-tilable by local extra-tilability of $\mathcal{E}$.

The link of a codimension two simplex of the universal development $\tilde{\mathscr{E}}$ of $\mathscr{E}$ lying over $\sigma$ is constructed as follows (cf. Corollary 18.6 in [JS1]). In the tree $\widetilde{\mathscr{E}}^{\sigma}$ (the universal development of $\left.\mathscr{\ell}^{\sigma}\right)$ take a ball $B=B_{k}\left(\left[\Delta_{\sigma}, 1\right], \widetilde{\mathscr{\vartheta}^{\sigma}}\right)$ of radius $k=6$. Let $H_{B}$ be the subgroup of the direct limit $\widehat{\mathscr{G}}^{\sigma}$ of $\mathscr{E}^{\sigma}$ for which $B$ is a strict fundamental domain. Such a subgroup exists by Proposition 2.7. The 1-dimensional link we look for is of the form $N \backslash \widetilde{\mathscr{G}^{\sigma}}$, for some finite index normal subgroup $N$ of $H(B)$. The image $p(B) \subset N \backslash \widetilde{\mathscr{\varphi}^{\sigma}}$ of $B$ is a strict fundamental domain for the action of a finite group $H_{B} / N$ on $N \backslash \widetilde{\mathscr{\ell}^{\sigma}}$, where $p: \widetilde{\mathscr{\vartheta}^{\sigma}} \rightarrow N \backslash \widetilde{\mathscr{\ell}^{\sigma}}$ is the quotient map.

We show that $A:=\left(N \backslash \widetilde{\mathscr{g}^{\sigma}}\right) \backslash p\left(\stackrel{\circ}{B}_{2}\left(\left[\Delta_{\sigma}, 1\right], \widetilde{\mathscr{g}^{\sigma}}\right)\right)$ is connected. Then $\left(N \backslash \widetilde{\mathscr{g}^{\sigma}}\right) \backslash$ $p\left(\stackrel{\circ}{B}_{2}\left(\left[\Delta_{\sigma}, g\right], \widetilde{\mathscr{E}^{\sigma}}\right)\right)$ is connected for every $g \in \widehat{\mathscr{\ell}^{\sigma}}$ since $N \backslash \widehat{\mathscr{\ell}^{\sigma}}$ acts transitively on 
the set of top-simplices of the link $N \backslash \widetilde{\mathscr{g}^{\sigma}}$. We show that every two points $x, y \in A$ can be connected by a path in $A$. Consider first the case when $x \in A \backslash p(B)$ and $y \in A \cap p(B)$. Consider the Cayley graph $\left(H_{B} / N, S\right)$ where $S$ is the set of generators defined as follows

$$
s \in S \quad \text { if and only if } \quad p(B) \cap s(p(B)) \neq \emptyset .
$$

This set generates $H_{B} / N$ due to connectedness of $B$ and $N \backslash \widetilde{\mathscr{g}^{\sigma}}$. Find a path in $A \cap p(B)$ joining $y$ with $y_{0} \in p(\partial B)$. Let $1 \neq s \in T$ be such that $y_{0} \in s(p(B))$. It is a general easy fact that the complement of a vertex (in particular, the complement of the vertex corresponding to 1) in any Cayley graph of a finite group is connected. Hence if $k \in H_{B} / N$ is such that $x \in k(p(B))$ we can find a sequence $s, s_{1}, \ldots, s_{l} \in S$ such that $s s_{1} \ldots s_{l}=k$ and $s s_{1} \ldots s_{i} \neq 1$, for $i=1,2, \ldots, l$. Then $s s_{1} \ldots s_{i}(p(B)) \subset$ $A$ for $i=1,2, \ldots, l$. For $i=1,2, \ldots, l-1$ and $y_{i}=s s_{1} \ldots s_{i-1}(p(B)) \cap$ $s s_{1} \ldots s_{i}(p(B))$ we can find a path in $s s_{1} \ldots s_{i-1}(p(B))$ connecting $y_{i-1}$ and $y_{i}$ for $i<l$ and a path in $k(p(B))$ connecting $y_{l-1}$ and $x$. Concatenation of those paths is a path in $A$ connecting $x$ and $y$. If $x, y \in A \cap p(B)$, then we can find a vertex $z \in A \backslash p(B)$ and connect $x$ and $y$ with $z$.

Similarly for $x, y \in A \backslash p(B)$. Thus $A$ is connected.

The proof that $\widetilde{\mathscr{E} \sigma} / N \backslash p\left(\dot{B}_{2}([z, 1], \widetilde{\mathscr{E} \sigma})\right)$ is connected for a vertex $z$ of $\Delta_{\sigma}$ is similar.

\section{References}

[BMcCM] N. Brady, J. McCammond, and J. Meier, Local-to-asymptotic topology for cocompact CAT(0) complexes. Topology Appl. 131 (2003), 177-188. Zbl 1045.20037 MR 1981872

[BriH] M. R. Bridson and A. Haefliger, Metric spaces of non-positive curvature. Grundlehren Math.Wiss. 319, Springer-Verlag, Berlin 1999. Zbl 0988.53001 MR 1744486

[Bro1] K. S. Brown, Cohomology of groups. Grad. Texts in Math. 87, Springer-Verlag, New York 1982. Zbl 0584.20036 MR 0672956

[Bro2] K. S. Brown, Buildings. Springer-Verlag, New York 1989. Zbl 0715.20017 MR 0969123

[D] M. W. Davis, Groups generated by reflections and aspherical manifolds not covered by Euclidean space. Ann. of Math. (2) 117 (1983), 293-324. Zbl 0531.57041 MR 690848

[DM] M. W. Davis and J. Meier, The topology at infinity of Coxeter groups and buildings. Comment. Math. Helv. 77 (2002), 746-766. Zbl 1022.20016 MR 1949112

[G] R. Geoghegan, Topological methods in group theory. Book in preparation. 
[GMi1] R. Geoghegan and M. L. Mihalik, Free abelian cohomology of groups and ends of universal covers. J. Pure Appl. Algebra 36 (1985), 123-137. Zbl 0577.20024 MR 787167

[GMi2] R. Geoghegan and M. L. Mihalik, A note on the vanishing of $H^{n}(G, \mathbb{Z} G)$. J. Pure Appl. Algebra 39 (1986), 301-304. Zbl 0599.20083 MR 821894

[H] F. Haglund, Complexes simpliciaux hyperboliques de grande dimension. Preprint, Prepublication Orsay 71, 2003.

[JS1] T. Januszkiewicz and J. Świątkowski, Simplicial nonpositive curvature. Publ. Math. Inst. Hautes Études Sci. 104 (2006), 1-85. Zbl 05117094 MR 2264834

[JS2] T. Januszkiewicz and J. Świątkowski, Filling invariants in systolic complexes and groups. Geom. Topol., to appear

[KS] R. C. Kirby and L. C. Siebenmann, On the triangulation of manifolds and the Hauptvermutung. Bull. Amer. Math. Soc. 75 (1969), 742-749. Zbl 0189.54701 MR 0242166

[O] D. Osajda, Ideal boundary of 7-systolic complexes and groups. Preprint.

[S] J. R. Stallings, On torsion-free groups with infinitely many ends. Ann. of Math. (2) 88 (1968), 312-334. Zbl 0238.20036 MR 0228573

[W] D. T. Wise, Sixtolic complexes and their fundamental groups. In preparation.

Received June 5, 2006; revised September 6, 2006

Instytut Matematyczny, Uniwersytet Wrocławski, pl. Grunwaldzki 2/4, 50-384 Wrocław, Poland

Institut de Mathématiques de Jussieu, Université Paris 6, Case 247, 4 Place Jussieu, 75252

Paris Cedex 05, France

E-mail: dosaj@math.uni.wroc.pl 